\title{
Local solvability for a class of differential complexes
}

\author{
by \\ PAULO D. CORDARO \\ and \\ JORGE G. HOUNIE \\ University of São Paulo \\ São Paulo, SP, Brazil \\ Federal University of São Carlos \\ São Carlos, SP, Brazil
}

\section{Introduction-The Treves conjecture}

In $1983 \mathrm{~F}$. Treves [T1] initiated the study of the local solvability for the class of differential complexes defined by a smooth, locally integrable structure of rank $n$ in $\mathbf{R}^{n+1}$. If $Z$ denotes a local first integral of the structure, Treves conjectured that the vanishing of the local cohomology in degree $q$ of such a differential complex would be related to the vanishing of the singular homology of the sets $Z=$ const. in dimension $q-1$. It is the purpose of this article to complete the proof of this conjecture in its full generality.

(A) In order to motivate and state the problem more precisely we first recall the question of local solvability for a single vector field in two variables. Let thus

$$
\mathrm{L}=a(x, t) \frac{\partial}{\partial t}+b(x, t) \frac{\partial}{\partial x}
$$

be a complex vector field defined in a neighborhood $X$ of the origin in $\mathbf{R}^{2}$ with no singularities. We say that $\mathrm{L}$ is solvable (at the origin) if the induced map $\mathrm{L}: \mathcal{C}_{0}^{\infty} \rightarrow \mathcal{C}_{0}^{\infty}$ is surjective, where we have written $\mathcal{C}_{0}^{\infty}$ to denote the space of germs of smooth functions at the origin.

The solvability of $\mathrm{L}$ is characterized by the so-called condition $(\mathcal{P})$ of NirenbergTreves (cf. [H, Chapter XXVI], [NT]). For the purpose of our presentation it is convenient to make an extra assumption and assume the integrability of $\mathrm{L}$, in the sense that there exists $Z \in \mathcal{C}^{\infty}(X)$ such that $\mathrm{L} Z=0$ and $\mathrm{d} Z \neq 0$ at every point of $X$.

It takes an elementary argument to show that we can choose such a solution $Z$ that can be written, in an appropriate coordinate system around the origin, as

$$
Z(x, t)=x+i \varphi(x, t)
$$

Both authors were partially supported by CNPq and FINEP. 
where $\varphi$ is smooth, real-valued and satisfies

$$
\varphi(0,0)=\varphi_{x}(0,0)=0 .
$$

In particular, we obtain that $\mathrm{L}$ is a non-vanishing multiple of the vector field

$$
\frac{\partial}{\partial t}-i \frac{\varphi_{t}}{1+i \varphi_{x}} \frac{\partial}{\partial x}
$$

and consequently its solvability at the origin is equivalent to the solvability at the origin of $(0.1)$.

Assume now that $X$ is a small rectangle centered at the origin and with sides parallel to the coordinate axes. Within this set-up the Nirenberg-Treves condition $(\mathcal{P})$ (cf. [NT]) can be stated as follows:

$$
t \mapsto \varphi(x, t) \text { is monotone, for all } x .
$$

Since for a fixed $z_{0}=x_{0}+i y_{0}$ the pre-image of the set $\left\{z_{0}\right\}$ by the map $Z$ is given by $\left\{\left(x_{0}, t\right): \varphi\left(x_{0}, t\right)=y_{0}\right\}$, it follows that $(0.2)$ is equivalent to:

$$
\left\{(x, t) \in X: Z(x, t)=z_{0}\right\} \text { is connected, for all } z_{0} .
$$

(B) We generalize the solvability problem just described taking as a starting point the special coordinates described in the preceding item. Let $a$ and $r$ be positive real numbers and set

$$
X=\left\{(x, t) \in \mathbf{R} \times \mathbf{R}^{n}:|x|<a,|t|<r\right\} .
$$

We take a smooth function

$$
Z(x, t)=x+i \varphi(x, t), \quad(x, t) \in X
$$

where $\varphi$ is a real-valued, smooth function satisfying

$$
\varphi(0,0)=\varphi_{x}(0,0)=0
$$

The orthogonal of $\{\mathrm{d} Z\}$ is spanned by the pairwise commuting vector fields

$$
\mathrm{L}_{j}=\frac{\partial}{\partial t_{j}}-i \frac{\varphi_{t_{j}}}{1+i \varphi_{x}} \frac{\partial}{\partial x}, \quad j=1, \ldots, n
$$

We introduce the space of differential forms on the open set $Y \subset X$,

$$
\mathcal{C}^{\infty}\left(Y, \bigwedge^{p}\right) \doteq\left\{u=\sum_{|J|=p} u_{J}(x, t) \mathrm{d} t_{J}, u_{J} \in \mathcal{C}^{\infty}(Y)\right\},
$$


and further introduce the differential complex

$$
\mathrm{L}: \mathcal{C}^{\infty}\left(Y, \bigwedge^{p}\right) \rightarrow \mathcal{C}^{\infty}\left(Y, \bigwedge^{p+1}\right)
$$

defined by

$$
\mathrm{L}(u)=\sum_{|J|=p} \sum_{j=1}^{n}\left(\mathrm{~L}_{j} u_{J}\right) \mathrm{d} t_{j} \wedge \mathrm{d} t_{J}
$$

(the fact that $\mathrm{L}^{2}=0$ follows from the relations $\left[L_{j}, L_{k}\right]=0$ ). We are now ready to generalize the previously discussed local solvability concept:

Definition 0.1 . The operator $\mathrm{L}$ is said to be solvable at the origin in degree $q$ $(1 \leqslant q \leqslant n)$ if for every $f \in \mathcal{C}_{0}^{\infty}\left(\Lambda^{q}\right), \mathrm{L} f=0$, there is $u \in \mathcal{C}_{0}^{\infty}\left(\bigwedge^{q-1}\right)$ such that $\mathrm{L} u=f$.

Here we are again denoting by $\mathcal{C}_{0}^{\infty}\left(\bigwedge^{r}\right)$ the space of germs $\lim _{Y \rightarrow\{0\}} \mathcal{C}^{\infty}\left(Y, \Lambda^{r}\right)$.

By an elementary category argument it is easily seen that this notion is equivalent to the following property:

$(\bullet)_{q}$ Given any open neighborhood $Y$ of the origin in $X$ there is another open neighborhood of the origin $Y^{\prime} \subset Y$ such that for every $f \in \mathcal{C}^{\infty}\left(Y, \wedge^{q}\right)$ satisfying $L f=0$ there is $u \in \mathcal{C}^{\infty}\left(Y^{\prime}, \bigwedge^{q-1}\right)$ such that $\mathrm{L} u=f$ in $Y^{\prime}$.

We notice some special cases. When $n=1$ there is only one operator and we get the situation already discussed in (A). Solvability in degree one means to solve the overdetermined system $\mathrm{L}_{j} u=f_{j}$ under the compatibility conditions $\mathrm{L}_{j} f_{k}=\mathrm{L}_{k} f_{j}$, whereas solvability in degree $n$ means to solve the underdetermined system $\mathrm{L}_{1} u_{1}+\ldots+\mathrm{L}_{n} u_{n_{n}}=f$ with no conditions on $f$ whatsoever.

(C) Invariance. We first recall that a complex line subbundle $\mathbf{T}^{\prime} \subset \mathrm{CT}^{*} X$ defines a locally integrable structure (in the sense of [T2]) if it is locally generated by the differential of a smooth function. To such a structure there is canonically associated a differential complex: it is simply the complex induced by the exterior derivative acting on the bundles $\Lambda^{q}\left(\mathbf{C T}^{*} U / \mathrm{T}^{\prime}\right)$. Let us then fix such a structure. In a neighborhood of the origin, $\mathrm{T}^{\prime}$ is spanned by the differential of a smooth function which can always be written, in an appropriate system of coordinates, as $Z(x, t)=x+i \varphi(x, t)$, where $\varphi$ has the properties described in the preceding item. The complex $\mathrm{L}$ is nothing else than the realization of the differential complex associated to $\mathrm{T}^{\prime}$, after choosing these local coordinates and $\left\{\mathrm{d} t_{J},|J|=q\right\}$ as a basis for the sections of the bundle $\wedge^{q}\left(\mathrm{CT}^{*} U / \mathrm{T}^{\prime}\right)$.

(D) If $z_{0} \in \mathbf{C}$ and $Y \subset X$ we will refer to the set $\mathcal{F}\left(z_{0}, Y\right)=\left\{(x, t) \in Y: Z(x, t)=z_{0}\right\}$ as a fiber of the map $Z: X \rightarrow \mathbf{C}$ over $Y$. The germs of such fibers at the origin are invariants of the locally integrable structure $T^{\prime}$, a fact that follows from the so-called Baouendi-Treves approximation formula $[\mathrm{BT}]$. We may now introduce the following property: 
$(*)_{q}$ Given any open neighborhood $Y$ of the origin in $X$ there is another open neighborhood of the origin $Y^{\prime} \subset Y$ such that, for every regular value $z_{0} \in \mathbf{C}$ of $Z: X \rightarrow \mathbf{C}$, either $\mathcal{F}\left(z_{0}, Y^{\prime}\right)=\varnothing$ or else the homomorphism

$$
\widetilde{H}_{q}\left(\mathcal{F}\left(z_{0}, Y^{\prime}\right), \mathbf{C}\right) \rightarrow \widetilde{H}_{q}\left(\mathcal{F}\left(z_{0}, Y\right), \mathbf{C}\right)
$$

induced by the inclusion map $\mathcal{F}\left(z_{0}, Y^{\prime}\right) \subset \mathcal{F}\left(z_{0}, Y\right)$ vanishes identically.

Here $\widetilde{H}_{*}(\cdot, \mathbf{C})$ denotes reduced homology with complex coefficients.

It was conjectured by $\mathrm{F}$. Treves in 1983 that solvability at the origin in degree $q$ for the complex L should be equivalent to the validity of property $(*)_{q-1}$. The particular case $n=1$ is of course valid, since $(*)_{0}$ is equivalent to condition $(\mathcal{P})$ according to our previous discussion.

Several articles have been published towards the verification of this conjecture. In [CT1] it is proved that $(\bullet)_{q} \Rightarrow(*)_{q-1}$ for all $q=1, \ldots, n$. Indeed, if $(*)_{q-1}$ does not hold, it is possible to violate an a priori inequality which, in turn, follows from $(\bullet)_{q}$ in a now familiar way introduced by L. Hörmander.

The reverse implication was proved for $q=1$ in [MT], and, for $q=n$, it follows from the arguments in [CH2]. The method of proof in this work owes a lot to the previous two papers: the approach of reducing the problem to the study of the boundary equations induced by an elliptic complex is taken from the former, whereas a key technical decomposition of closed forms is obtained from the Bochner formula already used in the latter.

If we furthermore make the extra assumption that the structure $T^{\prime}$ is real-analytic then the work $[\mathrm{ChT}]$ gives a complete proof that $(*)_{q-1} \Rightarrow(\bullet)_{q}$ for all $q=1, \ldots, n$, a result that had been previously proved in the cases $q=1$ [T1] and $q=n$ [CH1]. The basic idea for the proof in [ChT] is to study the de Rham equation on regular fibers and to analyze the size of the solution as regular fibers approach the singular set. That solutions with tempered growth can be obtained depends in an essential way on the analyticity assumptions.

We also mention that it is possible to give a meaning for hyperfunction solvability of the complex $L$ (which generalizes the natural concept within the real-analytic category), and to prove that condition $(*)_{q-1}$ is in fact equivalent to the hyperfunction solvability at the origin in degree $q$, for every $q=1, \ldots, n$. On this subject we refer to [CT2] and [CT3].

Summing up, in order to complete the proof of the conjecture, it remains to be proved that the implication $(*)_{q-1} \Rightarrow(\bullet)_{q}$ holds for $2 \leqslant q \leqslant n-1$ when $T^{\prime}$ is a smooth structure. The purpose of the present article is to present a complete proof of this statement, showing then the validity of the Treves conjecture in general: 
Theorem 0.1. Suppose that $Z \in \mathcal{C}^{\infty}$ and let $q \in\{1,2, \ldots, n\}$. Then condition $(*)_{q-1}$ implies the local solvability at the origin of the complex $\mathrm{L}$ in degree $q$.

In the work [MT] it is also proved that $(*)_{q-1}$ and $(*)_{q-2}$ together imply the local solvability of $\mathrm{L}$ in degree $q$ if $q \geqslant 2$. Some of the arguments of this article will be used in the proof of Theorem 0.1.

We shall organize our presentation as follows. In the first section we introduce the standard differential complex $\partial_{\bar{z}}+\mathrm{d}_{t}$ in $\mathbf{C} \times \mathbf{R}^{n}$, and apply a Mayer-Vietoris exact sequence argument to explain why our solvability problem is equivalent to the solvability of $\partial_{\bar{z}}+\mathrm{d}_{t}$ on both sides of a certain hypersurface constructed by means of the given function $Z$ (this is, of course, a similar device to the one used to study the solvability of the tangential Cauchy-Riemann complex in a hypersurface of the complex space). We will also follow an argument from $[\mathrm{MT}]$ in order to split the condition $(*)_{q-1}$ into two conditions $(*)_{q-1}^{+}$and $(*)_{q-1}^{-}$which will correspond to solvability on each side of the hypersurface.

In the remaining of the article we focus on the study of one-sided solvability on the side that corresponds to condition $(*)_{q-1}^{+}$, and present the full proof of the "micro-local" version of Theorem 0.1 (cf. Theorem 1.1 below). We have also found appropriate to present in an appendix a brief general discussion on condition $(*)_{q}^{+}$, where we do not pay attention to the growth of the solutions when approaching the hypersurface, but rather explain its meaning conceptually in terms of the partial de Rham operator $\mathrm{d}_{t}$ acting on $\mathbf{C} \times \mathbf{R}^{n}$. This sheds some light on the refinement of the argument in [MT] that is required to get the complete proof of the Treves conjecture.

\section{The elliptic complex in $\mathbf{C} \times \mathbf{R}^{n}$}

(A) We shall write the coordinates in the space $\mathbf{C} \times \mathbf{R}^{n}$ as $(z, t)=\left(z, t_{1}, \ldots, t_{n}\right)$, and if $\Omega \subset \mathbf{C} \times \mathbf{R}^{n}$ is open we shall denote by $\mathcal{C}_{(0, p)}^{\infty}(\Omega)$ the space of smooth differential forms on $\Omega$ of the kind

$$
f=\sum_{|K|=p-1} f_{K}(z, t) \mathrm{d} \bar{z} \wedge \mathrm{d} t_{K}+\sum_{|J|=p} f_{J}(z, t) \mathrm{d} t_{J}
$$

We shall also set

$$
\mathcal{C}^{\infty}\left(\Omega, \bigwedge^{0, p}\right)=\left\{f=\sum_{|J|=p} f_{J}(z, t) \mathrm{d} t_{J}: f_{J} \in \mathcal{C}^{\infty}(\Omega)\right\}
$$

and

$$
\mathcal{R}\left(\Omega, \bigwedge^{0, p}\right)=\left\{f \in \mathcal{C}^{\infty}\left(\Omega, \bigwedge^{0, p}\right): \partial_{\bar{z}} f=0\right\}
$$


We have the standard elliptic differential complex

$$
\partial_{\bar{z}}+\mathrm{d}_{t}: \mathcal{C}_{(0, p)}^{\infty}(\Omega) \rightarrow \mathcal{C}_{(0, p+1)}^{\infty}(\Omega)
$$

defined in the standard way, as well as its subcomplex

$$
\mathrm{d}_{t}: \mathcal{R}\left(\Omega, \bigwedge^{0, p}\right) \rightarrow \mathcal{R}\left(\Omega, \bigwedge^{0, p+1}\right) .
$$

Finally we denote by $H^{0, *}(\Omega)$ (resp. $H_{\mathrm{r}}^{0, *}(\Omega)$ ) the cohomology of the complex (1.4) (resp. (1.5)). A standard (and easy) argument allows one to prove (cf. [CT3, Lemma 3.4])

LEMma 1.1. If $\Omega$ is $(\partial / \partial \bar{z})$-convex (i.e. if $\partial / \partial \bar{z}$ defines a surjective endomorphism of $\mathcal{C}^{\infty}(\Omega)$ ) then the natural maps

$$
H_{\mathrm{r}}^{0, p}(\Omega) \rightarrow H^{0, p}(\Omega)
$$

are isomorphisms for all $p=0, \ldots n$.

(B) Now we return to the complex L given by $(0.8),(0.9)$. Taking advantage of the special form of the map $Z$ defined in (0.4), and also of the properties in (0.5), it is easy to see that if $X$ is sufficiently contracted about the origin, the set

$$
\Sigma \doteq\{(Z(x, t), t):(x, t) \in X\}
$$

defines a hypersurface in $\mathbf{C} \times \mathbf{R}^{n}$ containing the origin. Moreover, the complex $\partial_{\bar{z}}+\mathrm{d}_{t}$ induces a tangential complex on $\Sigma$ which is nothing else than the complex $\mathrm{L}$ after making the identification $X \simeq \Sigma$ via the map $(x, t) \mapsto(Z(x, t), t)$ (cf. [T2, $\S \S I .1, \mathrm{~V} .2]$ ). Let us now assume that $\omega$ is an open neighborhood of the origin in $\mathbf{C} \times \mathbf{R}^{n}$. We set

$$
\begin{aligned}
& \omega^{+} \doteq\{(z, t) \in \omega: z=x+i y, y>\varphi(x, t)\} \\
& \omega^{-} \doteq\{(z, t) \in \omega: z=x+i y, y<\varphi(x, t)\}, \\
& \overline{\omega^{ \pm}} \doteq \omega^{ \pm} \cup(\omega \cap \Sigma) .
\end{aligned}
$$

If we moreover denote by $H^{*}(\omega \cap \Sigma, \mathrm{L})$ the cohomology of the complex (0.8) over the open set $X \supset Y \simeq \omega \cap \Sigma$, we can apply [T2, Theorem V.3.1] and obtain the Mayer-Vietoris exact sequence

$$
\begin{aligned}
\ldots & \rightarrow \lim _{\omega \rightarrow 0} H^{0, p}(\omega) \rightarrow \lim _{\omega \rightarrow 0} H^{0, p}\left(\overline{\omega^{+}}\right) \oplus \lim _{\omega \rightarrow 0} H^{0, p}\left(\overline{\omega^{-}}\right) \rightarrow \\
& \rightarrow \lim _{\omega \rightarrow 0} H^{p}(\omega \cap \Sigma, \mathrm{L}) \rightarrow \lim _{\omega \rightarrow 0} H^{0, p+1}(\omega) \rightarrow \ldots,
\end{aligned}
$$

from which we conclude (notice that (1.4) is locally exact) 
Proposition 1.1. The operator $\mathrm{L}$ is solvable in degree $q$ if and only if

$$
\lim _{\omega \rightarrow 0} H^{0, q}\left(\overline{\omega^{ \pm}}\right)=0 .
$$

(C) The preceding proposition decomposes the solvability problem for the nonelliptic complex (0.8) into two problems involving an elliptic complex, thus shifting the obstruction to the geometry of the hypersurface $\Sigma$. In order to deal with these two problems separately we must decompose the solvability condition $(*)_{q}$, and for this we follow [MT]. Without loss of generality we can assume that $X$ contains the closure of

$$
Q=\left\{(x, t) \in \mathbf{R} \times \mathbf{R}^{n}:|x|<1,|t|<1\right\}
$$

Given $\sigma \in] 0,1\left[\right.$ and $z_{0}=x_{0}+y_{0} \in \mathbf{C}$ we let $\mathcal{F}_{-}\left(z_{0}\right)$ (the sublevel set) be defined by

$$
\mathcal{F}_{-}\left(z_{0}, \sigma\right) \doteq\left\{t:|t|<\sigma, \varphi\left(x_{0}, t\right)<y_{0}\right\}
$$

and introduce

$(*)_{q}^{+}$Given $\left.\sigma \in\right] 0,1\left[\right.$ there is $\left.\sigma^{\prime} \in\right] 0, \sigma\left[\right.$ such that the following is true for any $z_{0}$ :

(1) if $q=0$, every smooth and closed 0 -form on $\mathcal{F}_{-}\left(z_{0}, \sigma\right)$ is constant on $\mathcal{F}_{-}\left(z_{0}, \sigma^{\prime}\right)$,

(2) if $q \geqslant 1$, every smooth and closed $q$-form on $\mathcal{F}_{-}\left(z_{0}, \sigma\right)$ is exact on $\mathcal{F}_{-}\left(z_{0}, \sigma^{\prime}\right)$.

Likewise we can introduce condition $(*)_{q}^{-}$. We prove

LEMMA 1.2. $(*)_{q} \Rightarrow(*)_{q}^{+}$and $(*)_{q}^{-}$.

Proof. Let $\sigma \in] 0,1\left[\right.$ be fixed. According to $(*)_{q}$ there is $\left.\sigma^{\prime} \in\right] 0, \sigma[$ such that

$$
\widetilde{H}_{q}\left(\left\{t:|t|<\sigma^{\prime}, \varphi\left(x_{0}, t\right)=y_{0}\right\}, \mathbf{C}\right) \rightarrow \widetilde{H}_{q}\left(\left\{t:|t|<\sigma, \varphi\left(x_{0}, t\right)=y_{0}\right\}, \mathbf{C}\right)
$$

is trivial for every regular value $z_{0}=x_{0}+i y_{0}$.

According to [Br, II.10.3] there are exact sequences for cohomology with compact supports and coefficients in the constant sheaf $\mathbf{C}$

$$
\begin{aligned}
\ldots & \rightarrow H_{c}^{p}\left(\left\{t:|t|<\varrho, \varphi\left(x_{0}, t\right) \neq y_{0}\right\}, \mathbf{C}\right) \rightarrow H_{c}^{p}(\{t:|t|<\varrho\}, \mathbf{C}) \rightarrow \\
& \rightarrow H_{c}^{p}\left(\left\{t:|t|<\varrho, \varphi\left(x_{0}, t\right)=y_{0}\right\}, \mathbf{C}\right) \rightarrow \ldots
\end{aligned}
$$

which are natural in $\varrho \in] 0,1[$. Taking this into account together with the fact that

$$
H_{c}^{p}\left(\left\{t:|t|<\varrho, \varphi\left(x_{0}, t\right)=y_{0}\right\}, \mathbf{C}\right) \simeq H_{n-p}\left(\left\{t:|t|<\varrho, \varphi\left(x_{0}, t\right)=y_{0}\right\}, \mathbf{C}\right),
$$

we conclude the existence of natural isomorphisms

$$
\widetilde{H}_{q}\left(\left\{t:|t|<\varrho, \varphi\left(x_{0}, t\right)=y_{0}\right\}, \mathbf{C}\right) \simeq \widetilde{H}_{q}\left(\left\{t:|t|<\varrho, \varphi\left(x_{0}, t\right) \neq y_{0}\right\}, \mathbf{C}\right) .
$$


Since $(\sharp)$ is trivial we obtain in particular that

$$
\widetilde{H}_{q}\left(\mathcal{F}_{-}\left(z_{0}, \sigma^{\prime}\right), \mathbf{C}\right) \rightarrow \widetilde{H}_{q}\left(\mathcal{F}_{-}\left(z_{0}, \sigma\right), \mathbf{C}\right)
$$

also vanishes for all such $z_{0}$, and then for arbitrary $y_{0}$ by Sard's theorem, as an easy continuity argument for compactly supported cohomology shows. By de Rham's isomorphism it follows that $(*)_{q}^{+}$holds true. The other implication is analogous.

By Lemma 1.1 it follows that Theorem 0.1 will be a consequence of the implications

$$
\begin{aligned}
& (*)_{q-1}^{+} \Rightarrow \lim _{\omega \rightarrow 0} H^{0, q}\left(\overline{\omega^{+}}\right)=0, \\
& (*)_{q-1}^{-} \Rightarrow \lim _{\omega \rightarrow 0} H^{0, q}\left(\overline{\omega^{-}}\right)=0 .
\end{aligned}
$$

In the remaining of the paper we shall concentrate in the proof of $\left(1.14^{+}\right)$, the proof of $\left(1.14^{-}\right)$being of course analogous. $\left({ }^{1}\right)$ We will now make a more precise statement.

(D) We recall that we are given a smooth $\varphi(x, t)$ defined in a neighborhood of the closure of $Q$. Moreover, thanks to (0.5), we can assume the validity of the properties

$$
\begin{gathered}
\left|\varphi(x, t)-\varphi\left(x^{\prime}, t\right)\right| \leqslant \frac{1}{4}\left|x-x^{\prime}\right|, \quad \cdot(x, t),\left(x^{\prime}, t\right) \in Q, \\
|\varphi(x, t)| \leqslant C\left(|x|^{2}+|t|\right), \quad(x, t) \in Q .
\end{gathered}
$$

We shall also introduce the notation

$$
\begin{aligned}
& \Omega \doteq\left\{(x+i y, t) \in \mathbf{C} \times \mathbf{R}^{n}:|x|<1,|t|<1, \varphi(x, t)<y\right\}, \\
& \bar{\Omega} \doteq\left\{(x+i y, t) \in \mathbf{C} \times \mathbf{R}^{n}:|x|<1,|t|<1, \varphi(x, t) \leqslant y\right\},
\end{aligned}
$$

as well as we will denote by $\vartheta$ the set of all neighborhoods of the origin in $\mathbf{C} \times \mathbf{R}^{n}$ of the form $\Delta=R \times \Theta$, where $R$ (resp. $\Theta$ ) is an open rectangle with sides parallel to the coordinate axes (resp. a ball) centered at the origin in $\mathbf{C}$ (resp. $\mathbf{R}^{n}$ ).

The following theorem may be considered the main result of this work:

THEOREM 1.1. Assume that condition $(*)_{q-1}^{+}$holds. Then given $\Delta \in \vartheta$ there is $\Delta^{\prime} \in \vartheta, \Delta^{\prime} \subset \Delta$ such that the following is true:

Given $F \in \mathcal{R}\left(\bar{\Omega} \cap \Delta, \bigwedge^{0, q}\right)$ with $\mathrm{d}_{t} F=0$ there is $U \in \mathcal{R}\left(\bar{\Omega} \cap \Delta^{\prime}, \bigwedge^{0, q-1}\right)$ so that $\mathrm{d}_{t} U=F$ in $\Omega \cap \Delta^{\prime}$.

Remark. This statement shows that

$$
(*)_{q-1}^{+} \Rightarrow \lim _{\omega \rightarrow 0} H_{\mathrm{r}}^{0, q}\left(\overline{\omega^{+}}\right)=0,
$$

$\left({ }^{1}\right)$ In an unpublished manuscript by $\mathrm{F}$. Treves and the first author, the reverse implications in $\left(1.14^{+}\right)$and $\left(1.14^{-}\right)$are also proved to be true. 
and this immediately implies $\left(1.14^{+}\right)$, for, according to the argument that leads to the proof of Lemma 1.1, the surjectivity of $\partial / \partial \bar{z}: \lim _{\omega \rightarrow 0} \mathcal{C}^{\infty}\left(\overline{\omega^{+}}\right) \rightarrow \lim _{\omega \rightarrow 0} \mathcal{C}^{\infty}\left(\overline{\omega^{+}}\right)$shows that the natural map

$$
\lim _{\omega \rightarrow 0} H_{\mathrm{r}}^{0, q}\left(\overline{\omega^{+}}\right) \rightarrow \lim _{\omega \rightarrow 0} H^{0, q}\left(\overline{\omega^{+}}\right)
$$

is an isomorphism.

\section{Proof of Theorem 1.1-beginning}

(A) The Bochner formula. Let $\varepsilon>0, z \in \mathbf{C}$ and $k \in \mathbf{N}$ be fixed. We have

$$
\begin{aligned}
\int_{0}^{\infty} \mathrm{e}^{-\tau} \tau^{k-1} & \left(\int_{0}^{\infty} \mathrm{e}^{i z \xi-(\tau+\varepsilon) \xi^{2}} \mathrm{~d} \xi\right) \mathrm{d} \tau \\
& =\int_{0}^{\infty} \mathrm{e}^{-\tau} \tau^{k-1}\left(1-\partial_{\tau}\right)^{k} \underbrace{\left(\int_{0}^{\infty} \frac{1}{\left(1+\xi^{2}\right)^{k}} \mathrm{e}^{i z \xi-(\tau+\varepsilon) \xi^{2}} \mathrm{~d} \xi\right)}_{q_{k}(\tau)} \mathrm{d} \tau
\end{aligned}
$$

If we integrate this last expression by parts $k$ times, we conclude that its value equals $C_{k}^{-1} q_{k}(0)$ (where $C_{k}$ is a constant which depends only on $k$ ), and thus we obtain

$$
\int_{0}^{\infty} \frac{1}{\left(1+\xi^{2}\right)^{k}} \mathrm{e}^{i z \xi-\varepsilon \xi^{2}} \mathrm{~d} \xi=C_{k} \int_{0}^{\infty} \mathrm{e}^{-\tau} \tau^{k-1}\left(\int_{0}^{\infty} \mathrm{e}^{i z \xi-(\tau+\varepsilon) \xi^{2}} \mathrm{~d} \xi\right) \mathrm{d} \tau
$$

which can further be written, after the change of variables $\eta=\sqrt{\tau+\varepsilon} \xi$, as

$$
\int_{0}^{\infty} \frac{1}{\left(1+\xi^{2}\right)^{k}} \mathrm{e}^{i z \xi-\varepsilon \xi^{2}} \mathrm{~d} \xi=C_{k} \int_{0}^{\infty} \frac{\mathrm{e}^{-\tau} \tau^{k-1}}{(\tau+\varepsilon)^{1 / 2}}\left(\int_{0}^{\infty} \mathrm{e}^{i \eta z /(\tau+\varepsilon)^{1 / 2}-\eta^{2}} \mathrm{~d} \eta\right) \mathrm{d} \tau
$$

Remark. Notice that this formula is a generalization of the Bochner formula used in [CH2, cf. (1.9)]. Indeed, if the integration in $\xi$ is carried over all the real line, we would obtain (2.1) where the $\eta$-integral is also over $\mathbf{R}$. It suffices to use the identity

$$
\int_{\mathbf{R}} \mathrm{e}^{i z \eta-\eta^{2}} \mathrm{~d} \eta=\frac{1}{2 \sqrt{\pi}} \mathrm{e}^{-z^{2} / 4}
$$

(B) Let $F$ be as in the statement of Theorem 1.1. There is no loss of generality in assuming that all the derivatives of $F$ are bounded in $\bar{\Omega} \cap \Delta$. Moreover, thanks to (1.16), we can even assume that $\Delta=\{(z, t):|x|<r,|y|<s,|t|<\varrho\}$ is such that the following estimate holds:

$$
\|\varphi\|_{\Delta} \doteq \sup _{\Delta}|\varphi|<s<\frac{1}{32} r .
$$


We set

$$
F_{\varepsilon}(z, t)=\frac{1}{\pi} \int_{0}^{\infty} \int_{x^{\prime} \in \mathbf{R}} \mathrm{e}^{i \xi\left(z-Z\left(x^{\prime}, t\right)\right)-\varepsilon \xi^{2}} \psi\left(x^{\prime}\right) F\left(Z\left(x^{\prime}, t\right), t\right) \mathrm{d} Z\left(x^{\prime}, t\right) \mathrm{d} \xi,
$$

where $\psi \in \mathcal{C}_{c}^{\infty}(-r, r)$ is a cut-off function which is equal to one on $\left[-\frac{1}{2} r, \frac{1}{2} r\right]$. Of course, the following property is of the foremost importance:

LEMMA 2.1. $F_{\varepsilon}$ converges in $\mathcal{R}\left(\bar{\Omega}, \Lambda^{0, q}\right)$, as $\varepsilon \searrow 0$, to an element $F^{\bullet}$ which satisfies the property:

Setting $\Delta^{\bullet}=\left\{(z, t):|x|<\frac{1}{2} r,|y|<s,|t|<\varrho\right\}$, the restriction of $F-F^{\bullet}$ to $\Omega \cap \Delta^{\bullet}$ extends as an element of $\mathcal{R}\left(\Delta^{\bullet}, \Lambda^{0, q}\right)$.

Proof. The first statement is absolutely routine. For the second we introduce

$$
F_{\varepsilon}^{\sharp}(z, t)=\frac{1}{\pi} \int_{-\infty}^{0} \int_{x^{\prime} \in \mathbf{R}} \mathrm{e}^{i \xi\left(z-Z\left(x^{\prime}, t\right)\right)-\varepsilon \xi^{2}} \psi\left(x^{\prime}\right) F\left(Z\left(x^{\prime}, t\right), t\right) \mathrm{d} Z\left(x^{\prime}, t\right) \mathrm{d} \xi .
$$

Then $F_{\varepsilon}^{\sharp} \rightarrow F^{\sharp}$ in $\mathcal{R}\left(\overline{\Omega^{\sharp}}, \Lambda^{0, q}\right)$ as $\varepsilon \searrow 0$. Here we have written

$$
\overline{\Omega^{\sharp}} \doteq\left\{(x+i y, t) \in \mathbf{C} \times \mathbf{R}^{n}:|x|<1,|t|<1, \varphi(x, t) \geqslant y\right\} .
$$

Since we also have

$$
F(Z(x, t), t)-F^{\bullet}(Z(x, t), t)=F^{\sharp}(Z(x, t), t),
$$

when $|x|<\frac{1}{2} r,|t|<\varrho$, the result follows.

(C) We write $f(x, t) \doteq F(Z(x, t), t)$ and $g \doteq\left[1-M^{2}\right]^{k}(\psi f)$, where $M=Z_{x}^{-1} \partial / \partial x$, and observe that $\mathrm{L} g=0$ when $|x| \leqslant \frac{1}{2} r$, since $M$ and $\mathrm{L}$ commute and $\mathrm{L} f=0$. We make the usual integration by parts

$$
F_{\varepsilon}(z, t)=\frac{1}{\pi} \int_{0}^{\infty} \int_{x^{\prime} \in \mathbf{R}} \frac{1}{\left(1+\xi^{2}\right)^{k}} \mathrm{e}^{i \xi\left(z-Z\left(x^{\prime}, t\right)\right)-\varepsilon \xi^{2}} g\left(x^{\prime}, t\right) \mathrm{d} Z\left(x^{\prime}, t\right) \mathrm{d} \xi
$$

and apply our identity (2.1). We obtain

$$
F_{\varepsilon}(z, t)=\frac{C_{k}}{\pi} \int_{0}^{\infty} \mathrm{e}^{-\tau} \frac{\tau^{k-1}}{(\tau+\varepsilon)^{1 / 2}}\left(\int_{0}^{\infty} \int \mathrm{e}^{i \eta \frac{\left(z-Z\left(x^{\prime}, t\right)\right)}{(\tau+\varepsilon)^{1 / 2}}-\eta^{2}} g\left(x^{\prime}, t\right) \mathrm{d} Z\left(x^{\prime}, t\right) \mathrm{d} \eta\right) \mathrm{d} \tau
$$

We shall then make the dyadic decomposition

$$
\begin{aligned}
& F_{\varepsilon, p}(z, t) \\
& \quad \doteq \frac{C_{k}}{\pi} \int_{2^{-p-1}}^{2^{-p}} \mathrm{e}^{-\tau} \frac{\tau^{k-1}}{(\tau+\varepsilon)^{1 / 2}}\left(\int_{0}^{\infty} \int \mathrm{e}^{i \eta \frac{\left(z-Z\left(x^{\prime}, t\right)\right)}{(\tau+\varepsilon)^{1 / 2}}-\eta^{2}} g\left(x^{\prime}, t\right) \mathrm{d} Z\left(x^{\prime}, t\right) \mathrm{d} \eta\right) \mathrm{d} \tau \\
& F_{\varepsilon,-1}(z, t) \\
& \quad \doteq \frac{C_{k}}{\pi} \int_{1}^{\infty} \mathrm{e}^{-\tau} \frac{\tau^{k-1}}{(\tau+\varepsilon)^{1 / 2}}\left(\int_{0}^{\infty} \int \mathrm{e}^{i \eta \frac{\left(z-Z\left(x^{\prime}, t\right)\right)}{(\tau+\varepsilon)^{1 / 2}}-\eta^{2}} g\left(x^{\prime}, t\right) \mathrm{d} Z\left(x^{\prime}, t\right) \mathrm{d} \eta\right) \mathrm{d} \tau
\end{aligned}
$$


Thus

$$
F_{\varepsilon}=\sum_{p=0}^{\infty} F_{\varepsilon, p}+F_{\varepsilon,-1}
$$

In the sequel we shall use the notation

$$
\Omega_{p} \doteq\left\{(z, t):|x|<r,|t|<\varrho, \varphi(x, t)-2^{-p / 2}<y<s\right\} .
$$

Remark. The role of the decomposition (2.8) is as follows. The form $F_{\varepsilon}$ is an entire function of $z$, and we may estimate its size for $\varphi(x, t)-\varepsilon<y<s(|x|$ and $|t|$ small); so as $\varepsilon \rightarrow 0$ we may only control sizes uniformly in $\varepsilon$ on the set $\varphi(x, t) \leqslant y<s$. On the other hand, each term $F_{\varepsilon, p}$ in (2.8) may be controlled uniformly in $\varepsilon>0$ on the larger set $\Omega_{p}$, where it converges to a form $F_{p}$ which is holomorphic on both sides of the graph $y=\varphi(x, t)$. This is quantitatively expressed by the next lemma.

LEMMA 2.2. There are constants $C_{m, k}>0$ such that for every $p \in \mathbf{N}$ and every $\varepsilon>0$ we have

$$
\left\|F_{\varepsilon, p}\right\|_{m, \Omega_{p}} \doteq \sup _{\Omega_{p}} \sup _{|\alpha| \leqslant m}\left|\partial^{\alpha} F_{\varepsilon, p}\right| \leqslant C_{m, k} 2^{-(k-1-m) p} .
$$

Proof. We first consider the case $m=0$. In the definition of $F_{\varepsilon, p}$ perform first the integral in the $\eta$-variable. We fix $(z, t) \in \Omega_{p}$ and write $z=x+i(\varphi(x, t)+a)$ where $a>-2^{-p / 2}$. After we deform the $\eta$-integration to the chain

$$
\eta \mapsto \eta\left(1+\frac{i}{2} \frac{x-x^{\prime}}{\left|x-x^{\prime}\right|}\right)
$$

we get as the real part of the exponent the quantity

$$
\mathcal{Q}=\frac{1}{(\tau+\varepsilon)^{1 / 2}}\left(-\frac{1}{2}\left|x-x^{\prime}\right|+\varphi\left(x^{\prime}, t\right)-\varphi(x, t)-a\right) \eta-\frac{3}{4} \eta^{2} .
$$

From (1.15) we obtain

$$
\mathcal{Q} \leqslant \frac{-a}{(\tau+\varepsilon)^{1 / 2}} \eta-\frac{3}{4} \eta^{2} \leqslant \frac{2^{-p / 2}}{\tau^{1 / 2}} \eta-\frac{3}{4} \eta^{2} .
$$

Since in the domain of the $\tau$-integration in the definition of $F_{\varepsilon, p}^{+}$we have $\tau>2^{-p-1}$, we conclude that

$$
\mathcal{Q} \leqslant \sqrt{2} \eta-\frac{3}{4} \eta^{2}
$$

Hence we obtain

$$
\left\|F_{\varepsilon, p}\right\|_{0, \Omega_{p}} \leqslant C \int_{2^{-p-1}}^{2^{-p}} \tau^{k-3 / 2} \mathrm{~d} \tau .
$$

For the other values of $m$ the estimates follow from differentiation under the integral sign. 
LEMMA $2.2^{\prime}$. There are constants $C_{m, k}>0$ such that for every $\varepsilon>0$ we have

$$
\left\|F_{\varepsilon,-1}\right\|_{m, \Delta} \leqslant C_{m, k} .
$$

Proof. It follows immediately from derivation under the integral sign.

(D) Next we shall consider the action of the exterior derivative $\mathrm{d}_{t}$ in the expressions (2.6). If we apply [T2, Lemma II.2.2] and make the change of variables $\eta=\sqrt{\tau+\varepsilon} \xi$, we obtain

$$
\begin{aligned}
& \mathrm{d}_{t} F_{\varepsilon, p}(z, t) \\
& \quad=\frac{C_{k}}{\pi} \int_{2^{-p-1}}^{2^{-p}} \mathrm{e}^{-\tau} \tau^{k-1}\left(\int_{0}^{\infty} \int \mathrm{e}^{i \xi\left(z-Z\left(x^{\prime}, t\right)\right)-(\tau+\varepsilon) \xi^{2}} \mathrm{~L} g\left(x^{\prime}, t\right) \mathrm{d} Z\left(x^{\prime}, t\right) \mathrm{d} \xi\right) \mathrm{d} \tau .
\end{aligned}
$$

Here we again shift the $\xi$-integral to the chain

$$
\xi \mapsto \xi\left(1+\frac{i}{2} \frac{x-x^{\prime}}{\left|x-x^{\prime}\right|}\right),
$$

where we are writing $z=x+i y$. The real part of the exponent becomes

$$
\mathcal{Q}^{\prime}=\left(-\frac{1}{2}\left|x-x^{\prime}\right|+\varphi\left(x^{\prime}, t\right)+y\right) \eta-\frac{3}{4}(\tau+\varepsilon) \xi^{2} .
$$

Since in the $x^{\prime}$-integral we have $\left|x^{\prime}\right| \geqslant \frac{1}{2} r$, we obtain from 2.13 , for $|x|<\frac{1}{4} r$ and $|t|<\varrho$, and taking account of $(2.2)$, that

$$
\mathcal{Q}^{\prime} \leqslant\left(-\frac{1}{8} r+\|\varphi\|_{\Delta}+s\right) \xi \leqslant-\frac{1}{16} r \xi
$$

If we let

$$
\Delta^{\bullet} \doteq\left\{(x+i y, t):|x|<\frac{1}{4} r,|y|<s,|t|<\varrho\right\},
$$

inequality (2.14) allows us to state

LEMMA 2.3. There are constants $C_{m, k}>0$ such that for every $p \in \mathbf{N}$ and every $\varepsilon>0$ we have

$$
\left\|\mathrm{d}_{t} F_{\varepsilon, p}\right\|_{m, \Delta^{*}} \leqslant C_{m, k} 2^{-(k-1-m) p} .
$$

Our next step is to solve the equations

$$
\mathrm{d}_{t} G_{\varepsilon, p}=\mathrm{d}_{t} F_{\varepsilon, p} \quad \text { in } \Delta^{\bullet}
$$

with estimates

$$
\left\|G_{\varepsilon, p}\right\|_{m, \Delta^{*}} \leqslant C_{m, k} 2^{-(k-1-m) p} .
$$

To that extent we use Poincaré's lemma in the $t$-variables, obtaining a solution that depends holomorphically on $z$. We set

$$
H_{\varepsilon, p} \doteq F_{\varepsilon, p}-G_{\varepsilon, p}
$$

The forms $H_{\varepsilon, p}$ are of course $\mathrm{d}_{t}$-closed (and consequently $\mathrm{d}_{t}$-exact) in $\Delta^{*}$; moreover, Lemma 2.2 and (2.17) give 
Lemma 2.4. There are constants $C_{m, k}>0$ such that for $p \in \mathbf{N}$ and every $\varepsilon>0$ we have

$$
\begin{aligned}
\left\|H_{\varepsilon, p}\right\|_{m, \Omega_{p} \cap \Delta^{*}} & \leqslant C_{m, k} 2^{-(k-1-m) p} \\
\left\|H_{\varepsilon,-1}\right\|_{m, \Omega_{p} \cap \Delta^{*}} & \leqslant C_{m, k} .
\end{aligned}
$$

\section{The main step}

(A) Before we proceed we pause to discuss a crucial tool that will be borrowed from [MT]. For this we fix $\Delta_{0} \in \vartheta$,

$$
\Delta_{0}=\left\{(z, t):|x|<r_{0},|y|<s_{0},|t|<\varrho_{0}\right\}
$$

and for $\delta>0$ also fixed we introduce the Lipschitz-continuous function

$$
\lambda_{\Delta_{0}, \delta}(z, t)=\min \left\{y-\varphi(x, t)+\delta, r_{0}-|x|, s_{0}-|y|, \varrho_{0}-|t|\right\} .
$$

Notice that there is a Lipschitz constant $C>0$ for $\lambda_{\Delta_{0}, \delta}$ which depends only on $\varphi$. We shall also set, for $\mu \in \mathbf{Z}_{+}$,

$$
\begin{aligned}
\mathcal{O}_{\delta} & =\left\{(z, t): \lambda_{\Delta_{0}, \delta}(z, t)>0\right\}, \\
\mathcal{O}_{\delta, \mu} & =\left\{(z, t): \lambda_{\Delta_{0}, \delta}(z, t)>C \sqrt{n} / 2^{\mu}\right\} .
\end{aligned}
$$

Finally, for a subset $A$ of $\mathbf{C} \times \mathbf{R}^{n}$ we shall write $A(z)$ to denote the slice $\left\{t \in \mathbf{R}^{n}:(z, t) \in A\right\}$.

Proposition 3.1 (cf. [MT, Lemma 4.1]). There exist constants $C_{m}>0$ such that the following is true: given any $S \in \mathcal{C}^{\infty}\left(\mathcal{O}_{\delta}, \bigwedge^{0, j}\right)$ whose restriction to $\{z\} \times \mathcal{O}_{\delta}(z)$ is $\mathrm{d}_{t^{-}}$ exact for any $z$, then for any $\mu$ so that $\mathcal{O}_{\delta, \mu} \neq \varnothing$ there exists $u_{\mu} \in \mathcal{C}^{\infty}\left(\mathcal{O}_{\delta, \mu}, \bigwedge^{0, j-1}\right)$ such that $\mathrm{d}_{t} u_{\mu}=S$ in $\mathcal{O}_{\delta, \mu}$ and

$$
\left\|u_{\mu}\right\|_{m, \mathcal{O}_{\delta, \mu}} \leqslant C_{m} 2^{(m+n+1) \mu}\|S\|_{m, \mathcal{O}_{\delta, \mu+2}} .
$$

(B) With this result in hand we can now state and prove the most important tool to be used in the proof of Theorem 2.1.

PRoposition 3.2. If condition $(*)_{q-1}^{+}$holds true then there exist $p_{0} \in \mathbf{N}$ and $\Delta_{1} \in \vartheta$ (both depending only on $\Delta), \Delta_{1} \subset \Delta^{*}$, and forms $V_{\varepsilon, p} \in \mathcal{R}\left(\Omega \cap \Delta_{1}, \bigwedge^{q-1}\right)\left(p \geqslant p_{0}\right)$ solving

$$
\mathrm{d}_{t} V_{\varepsilon, p}=H_{\varepsilon, p} \quad \text { in } \Omega \cap \Delta_{1}
$$


and satisfying

$$
\left\|V_{\varepsilon, p}\right\|_{m, \Omega \cap \Delta_{1}} \leqslant C_{m} 2^{(m+n+3) p}\left\|H_{\varepsilon, p}\right\|_{m+2, \Omega_{p} \cap \Delta^{*}}
$$

Proof. We start by applying Proposition 3.1 to the following choices: $\Delta_{0}=\Delta^{*}, \delta=$ $2^{-p / 2}$. Then $\mathcal{O}_{\delta}=\Omega_{p} \cap \Delta^{*}$, and consequently, since $H_{\varepsilon, p}$ is $\mathrm{d}_{t}$-exact on $\Delta^{*}$, we can find $u_{\varepsilon, p, \mu} \in \mathcal{C}^{\infty}\left(\mathcal{O}_{\delta, \mu}\right)$ such that

$$
\mathrm{d} u_{\varepsilon, p, \mu}=H_{\varepsilon, p} \quad \text { in } \mathcal{O}_{\delta, \mu}
$$

and

$$
\left\|u_{\varepsilon, p, \mu}\right\|_{m, \mathcal{O}_{\delta, \mu}} \leqslant C_{m} 2^{(m+n+1) \mu}\left\|H_{\varepsilon, p}\right\|_{m, \Omega_{p} \cap \Delta^{\bullet}} .
$$

Next we select $\Delta^{\bullet \bullet} \in \vartheta, \Delta^{\bullet \bullet} \subset \Delta^{\bullet}$ and $p_{0} \in \mathbf{N}$ such that

$$
(z, t) \in \Delta^{\bullet \bullet} \Rightarrow \lambda_{\Delta^{*}, 2^{-p / 2}}(z, t)=y-\varphi(x, t)+2^{-p / 2}
$$

for all $p \geqslant p_{0}$. Thus

$$
\mathcal{O}_{\delta, \mu} \cap \Delta^{* *}=\left\{(z, t) \in \Delta^{* *}: y>\varphi(x, t)+C \sqrt{n} 2^{-\mu}-2^{-p / 2}\right\}
$$

If we now take $\mu=\left[\frac{1}{2} p\right]+N$, with $N$ conveniently large but independent of $p$, in such a way that $\Omega_{p+2} \cap \Delta^{\bullet \bullet} \subset \mathcal{O}_{\delta, \mu} \cap \Delta^{\bullet \bullet}$, we can summarize:

For every $p \geqslant p_{0}$ and $\varepsilon>0$ there is $u_{\varepsilon, p} \in \mathcal{C}^{\infty}\left(\Omega_{p+2} \cap \Delta^{\bullet}, \bigwedge^{0, q-1}\right)$ such that

$$
\mathrm{d}_{t} u_{\varepsilon, p}=H_{\varepsilon, p} \quad \text { in } \Omega_{p+2} \cap \Delta^{\bullet \bullet}
$$

and

$$
\left\|u_{\varepsilon, p}\right\|_{m, \Omega_{p+2} \cap \Delta^{*}} \leqslant C_{m}^{\prime} 2^{(m+n+1) p / 2}\left\|H_{\varepsilon, p}\right\|_{m, \Omega_{p} \cap \Delta^{*}}
$$

Since $(\partial / \partial \bar{z}) H_{\varepsilon, p}=0$ we have $\mathrm{d}_{t}(\partial / \partial \bar{z}) u_{\varepsilon, p}=0$. We have now reached the point where we use property $(*)_{q-1}^{+}$. If we write $\Delta^{\bullet \bullet}=R^{\bullet \bullet} \times\left\{|t|<\varrho^{\bullet \bullet}\right\}$, it follows that

$$
\left(\Omega_{p+2} \cap \Delta^{\bullet \bullet}\right)(z)=\left\{t:|t|<\varrho^{\bullet \bullet}, y>\varphi(x, t)-2^{-(p+2) / 2}\right\} \quad(z=x+i y) .
$$

Hence by condition $(*)_{q-1}^{+}$there is $\Delta^{\dagger} \in \vartheta, \Delta^{\dagger} \subset \Delta^{\bullet}$, such that the following is true:

(a) If $q=1$, the restriction of $(\partial / \partial \bar{z}) u_{\varepsilon, p}$ to any slice $\{z\} \times\left(\Omega_{p+2} \cap \Delta^{\dagger}\right)(z)$ is constant;

(b) If $q \geqslant 2$, the restriction of $(\partial / \partial \bar{z}) u_{\varepsilon, p}$ to any slice $\{z\} \times\left(\Omega_{p+2} \cap \Delta^{\dagger}\right)(z)$ is exact.

To complete the proof we must now distinguish two cases.

Case 1: $q \geqslant 2$. We avail ourselves of (b). Applying the procedure described above to solve the equation

$$
\mathrm{d}_{t} \alpha=(\partial / \partial \bar{z}) u_{\varepsilon, p}
$$


we obtain $\Delta^{\dagger \dagger} \in \vartheta, \Delta^{\dagger \dagger} \subset \Delta^{\dagger}$, and, for an enlarged $p_{0}$, a sequence

$$
\alpha_{\varepsilon, p} \in \mathcal{C}^{\infty}\left(\Omega_{p+4} \cap \Delta^{\dagger \dagger}, \wedge^{0, q-2}\right) \quad\left(p \geqslant p_{0}\right)
$$

such that

$$
\mathrm{d}_{t} \alpha_{\varepsilon, p}=(\partial / \partial \bar{z}) u_{\varepsilon, p} \quad \text { in } \Omega_{p+4} \cap \Delta^{\dagger \dagger}
$$

and

$$
\left\|\alpha_{\varepsilon, p}\right\|_{m, \Omega_{p+4} \cap \Delta^{\dagger \dagger}} \leqslant C_{m}^{\prime \prime} 2^{(m+n+1) p / 2}\left\|(\partial / \partial \bar{z}) u_{\varepsilon, p}\right\|_{m, \Omega_{p+2} \cap \Delta^{\dagger}} .
$$

Finally we select $\chi_{p} \in \mathcal{C}^{\infty}(\mathbf{R})$ satisfying

$$
\begin{aligned}
& \chi_{p}(\tau)= \begin{cases}0 & \text { for } \tau<2^{-(p+6) / 2}, \\
1 & \text { for } \tau>2^{-(p+8) / 2},\end{cases} \\
& \left|\partial^{k} \chi_{p}\right| \leqslant C_{k} 2^{k p / 2},
\end{aligned}
$$

as well as $\gamma \in \mathcal{C}_{c}^{\infty}\left(R^{\dagger \dagger}\right)$ which is identically one in an open rectangle $R_{1} \subset R^{\dagger \dagger}$ centered at the origin and with sides parallel to the coordinate axes (where we are writing $\Delta^{\dagger \dagger}=$ $\left.R^{\dagger \dagger} \times\left\{|t|<\varrho^{\dagger \dagger}\right\}\right)$.

We set

$$
\tilde{\alpha}_{\varepsilon, p}(z, t) \doteq \gamma(z) \chi_{p}(y-\varphi(x, t)) \alpha_{\varepsilon, p}(z, t) .
$$

Observe that $\tilde{\alpha}_{\varepsilon, p}$ is defined in $\mathbf{C} \times\left\{|t|<\varrho^{\dagger \dagger}\right\}$, it has compact support in the $z$-variable and coincides with $\alpha_{\varepsilon, p}$ in $\Omega_{p+8} \cap \Delta_{1}$ if we set $\Delta_{1} \doteq R_{1} \times\left\{|t|<\varrho^{\dagger \dagger}\right\}$. From this we see that

$$
V_{\varepsilon, p} \doteq u_{\varepsilon, p}-\mathrm{d}_{t}\left[E * \tilde{\alpha}_{\varepsilon, p}\right]
$$

where $E(z)=1 / \pi z$ and the convolution is performed in the $z$-variable only, satisfies (cf. (3.11) and (3.13))

$$
\mathrm{d}_{t} V_{\varepsilon, p}=H_{\varepsilon, p}, \quad(\partial / \partial \bar{z}) V_{\varepsilon, p}=0 \quad \text { in } \Omega_{p+8} \cap \Delta_{1} .
$$

Moreover,

$$
\begin{aligned}
\left\|V_{\varepsilon, p}\right\|_{m, \Omega \cap \Delta_{1}} & \leqslant C_{m}^{(1)}\left[\left\|u_{\varepsilon, p}\right\|_{m, \Omega \cap \Delta_{1}}+\left\|E * \tilde{\alpha}_{\varepsilon, p}\right\|_{m+1, \Omega \cap \Delta_{1}}\right] \\
& \leqslant C_{m}^{(2)}\left[\left\|u_{\varepsilon, p}\right\|_{m, \Omega \cap \Delta_{1}}+\left\|\tilde{\alpha}_{\varepsilon, p}\right\|_{m+1, \Delta_{1}}\right]
\end{aligned}
$$

and then we can apply (3.12), (3.14) and (3.15) to obtain

$$
\begin{aligned}
& \left\|V_{\varepsilon, p}\right\|_{m, \Omega \cap \Delta_{1}}
\end{aligned}
$$

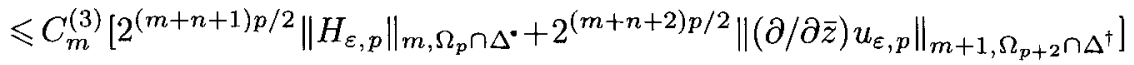

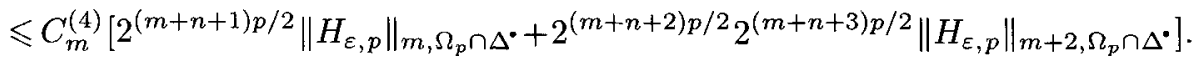

Consequently (3.6) holds and the proof is concluded in this case. 
Case 2: $q=1$. Let $\beta_{\varepsilon, p}$ be the function $(\partial / \partial \bar{z}) u_{\varepsilon, p}$ restricted to $\Omega_{p+2} \cap \Delta^{\dagger}$. By condition (a) above we know that $\beta_{\varepsilon, p}$ is independent of $t$. More precisely, if we denote by $\wp: \mathbf{C} \times \mathbf{R}^{n} \rightarrow \mathbf{C}$ the projection map, it follows that $\beta_{\varepsilon, p}$ is smooth in $\wp\left(\Omega_{p+2} \cap \Delta^{\dagger}\right)$ and satisfies the bounds

$$
\left\|\beta_{\varepsilon, p}\right\|_{m, \wp\left(\Omega_{p+2} \cap \Delta^{\dagger}\right)} \leqslant C_{m} 2^{(m+n+2) p / 2}\left\|H_{\varepsilon, p}\right\|_{m+1, \Omega_{p} \cap \Delta^{*}}
$$

Writing

$$
\Delta^{\dagger}=R^{\dagger} \times\left\{|t|<\varrho^{\dagger}\right\}=\left\{(z, t):|x|<r^{\dagger},|y|<s^{\dagger},|t|<\varrho^{\dagger}\right\}
$$

and

$$
\varphi^{*}(x) \doteq \inf \left\{\varphi(x, t):|t|<\varrho^{\dagger}\right\}
$$

it follows that

$$
\wp\left(\Omega_{p+2} \cap \Delta^{\dagger}\right)=\left\{z:|x|<r^{\dagger}, \varphi^{*}(x)-2^{-(p+2) / 2}<y<s^{\dagger}\right\}
$$

Let $R_{1} \subset \subset R^{\dagger}$ be an open rectangle centered at the origin and with sides parallel to the coordinate axes, and set $\Delta_{1} \doteq R_{1} \times\left\{|t|<\varrho^{\dagger}\right\}$. Next we select a cut-off $\eta_{p} \in \mathcal{C}_{c}^{\infty}\left(\wp\left(\Omega_{p+2} \cap \Delta^{\dagger}\right)\right)$ which is identically one in $\wp\left(\Omega_{p+4} \cap \Delta_{1}\right)$ and satisfies the bounds $\left({ }^{2}\right)$

$$
\left\|\partial^{k} \eta_{p}\right\|_{R^{\dagger}} \leqslant C_{k} 2^{|k| p / 2}
$$

Proceeding as in Case 1 it is easy to show that

$$
V_{\varepsilon, p} \doteq u_{\varepsilon, p}-E *\left(\eta_{k} \beta_{\varepsilon, p}\right)
$$

satisfies

$$
\mathrm{d}_{t} V_{\varepsilon, p}=H_{\varepsilon, p}, \quad(\partial / \partial \bar{z}) V_{\varepsilon, p}=0 \quad \text { in } \Omega_{p+2} \cap \Delta_{1}
$$

and, from (3.12), (3.19) and (3.20),

$$
\left\|V_{\varepsilon, p}\right\|_{m, \Omega \cap \Delta_{1}} \leqslant C_{m} 2^{(m+n+2) p / 2}\left\|H_{\varepsilon, p}\right\|_{m+1, \Omega_{p} \cap \Delta^{*}}
$$

This completes the proof of Proposition 3.2.

$\left({ }^{2}\right)$ Since $\varphi^{*}$ is only Lipschitz continuous, for the construction of $\eta_{p}$ we must recall some results on the regularized distance presented in $[\mathrm{S}$, Chapter VI]. In particular, we apply [S, Lemma 2 on p. 182]. 


\section{Proof of Theorem 1.1 (conclusion)}

(A) Fix $l \in \mathbf{N}$. If we apply (2.19) and (3.6), we conclude that choosing $k$ large enough in comparison with $n$ and $l$ allows us to make the series

$$
V_{\varepsilon} \doteq \sum_{p \geqslant p_{0}} V_{\varepsilon, p}
$$

to be convergent in the $\mathcal{C}^{l+2}$-topology on $\Omega \cap \Delta_{1}(k=2 l+n+10$ does the job). Moreover, the same estimates show that

$$
\sup _{\varepsilon>0}\left\|V_{\varepsilon}\right\|_{l+2, \Omega \cap \Delta_{1}}<\infty .
$$

We have (cf. (2.8), (2.18) and (3.5))

$$
\mathrm{d}_{t} V_{\varepsilon}=F_{\varepsilon}-\left[\sum_{p} G_{\varepsilon, p}+\sum_{p=-1}^{p_{0}-1} F_{\varepsilon, p}\right] \quad \text { in } \Omega \cap \Delta_{1} .
$$

Set

$$
\widetilde{G}_{\varepsilon} \doteq \sum_{p} G_{\varepsilon, p}+\sum_{p=-1}^{p_{0}-1} F_{\varepsilon, p} .
$$

Using Lemma $2.2^{\prime}$ in conjunction with (2.17) and an appropriate choice of $k$ we obtain

$$
\sup _{\varepsilon>0}\left\|\widetilde{G}_{\varepsilon}\right\|_{l+1, \Delta^{*}}<\infty
$$

By Ascoli's theorem we obtain forms

$$
V \in \mathcal{C}^{l}\left(\bar{\Omega} \cap \Delta_{1}, \bigwedge^{0, q-1}\right), \quad \widetilde{G} \in \mathcal{C}^{l}\left(\Delta_{1}, \bigwedge^{0, q}\right)
$$

such that

$$
\begin{aligned}
\mathrm{d}_{t} V & =F^{\bullet}-\widetilde{G} \quad \text { in } \Omega \cap \Delta_{1}, \\
(\partial / \partial \bar{z}) V & =0 \quad \text { in } \Omega \cap \Delta_{1}, \\
(\partial / \partial \bar{z}) \widetilde{G} & =0 \quad \text { in } \Delta_{1} .
\end{aligned}
$$

By Lemma 2.1 we can correct $\widetilde{G}$, maintaining of course the validity of (4.8), in such a way that now instead of (4.6) we have

$$
\mathrm{d}_{t} V=F-\widetilde{G} \quad \text { in } \Omega \cap \Delta_{1} .
$$

But then we have $\mathrm{d}_{t} \widetilde{G}=0$ in $\Omega \cap \Delta_{1}$, and consequently also in $\Delta_{1}$ by analytic continuation. If we finally solve

$$
\mathrm{d}_{t} W=\widetilde{G} \quad \text { in } \Delta_{1}
$$

where $W \in \mathcal{C}^{l}\left(\Delta_{1}, \Lambda^{0, q}\right)$ satisfies $(\partial / \partial \bar{z}) W=0$, then $U \doteq V-W \in \mathcal{C}^{l}\left(\bar{\Omega} \cap \Delta_{1}, \bigwedge^{0, q}\right)$ satisfies $\mathrm{d}_{t} U=F,(\partial / \partial \bar{z}) U=0$ in $\Omega \cap \Delta_{1}$. We summarize: 
Proposition 4.1. Assume that condition $(*)_{q-1}^{+}$holds. Then given $\Delta \in \vartheta$ there is $\Delta_{1} \in \vartheta, \Delta_{1} \subset \Delta$, such that the following is true:

Given $F \in \mathcal{R}\left(\bar{\Omega} \cap \Delta, \Lambda^{0, q}\right)$ with $\mathrm{d}_{t} F=0$ and $l \in \mathbf{N}$ there is $U_{l} \in \mathcal{C}^{l}\left(\bar{\Omega} \cap \Delta_{1}, \bigwedge^{0, q-1}\right)$ so that $\mathrm{d}_{t} U_{l}=F$ and $(\partial / \partial \bar{z}) U_{l}=0$ in $\Omega \cap \Delta_{1}$.

Remark. Recalling that $k$ is the number of times the second-order differential operator $\left[1-M^{2}\right]$ is applied to the trace of $F$ in $y=\varphi(x, t)$, we see that the choice $k=2 l+n+10$ shows that, roughly speaking, $\left[\frac{1}{2} n\right]+6$ derivatives are lost in the construction of $U$ in the sense that derivatives up to order $l$ of the trace of $U$ in a neighborhood of the origin may be estimated by the size of derivatives of the trace of $F$ of order $\leqslant l+\left[\frac{1}{2} n\right]+6$ in a larger neighborhood.

(B) In order to complete the proof of Theorem 1.1 we must show how to obtain smooth solutions from the statement in Proposition 4.1. The argument is of course standard provided a convenient approximation result for closed forms with holomorphic coefficients is available. We now pause to obtain such a result.

Proposition 4.2. Let $q \geqslant 1$ and $\Delta \in \vartheta$. Then there is $\Delta^{\prime} \in \vartheta, \Delta^{\prime} \subset \Delta$, such that the following is true, whatever $l \in \mathbf{N}$ :

Given $F \in \mathcal{C}^{l}\left(\bar{\Omega} \cap \Delta, \bigwedge^{0, q}\right)$ with $\mathrm{d}_{t} F=0$ and $(\partial / \partial \bar{z}) F=0$ there is a sequence $f_{\nu} \in$ $\mathrm{d}_{t} \mathcal{R}\left(\bar{\Omega} \cap \Delta^{\prime}, \Lambda^{0, q-1}\right)$ so that $f_{\nu} \rightarrow F$ in the $\mathcal{C}^{l-1}$-topology of $\bar{\Omega} \cap \Delta^{\prime}$.

Proof. We begin by recalling the so-called Baouendi-Treves approximation scheme. Let $\psi \in \mathcal{C}_{c}^{\infty}(\mathbf{R})$ be identically one in a small interval centered at the origin, and form

$$
F_{\nu}(z, t)=\left(\frac{\nu}{\pi}\right)^{1 / 2} \int \mathrm{e}^{-\nu\left(z-Z\left(x^{\prime}, t\right)\right)^{2}} \psi\left(x^{\prime}\right) F\left(Z\left(x^{\prime}, t\right), t\right) \mathrm{d} Z\left(x^{\prime}, t\right) .
$$

There is $\Delta_{1} \in \vartheta, \Delta_{1} \subset \Delta$, such that the following is true:

$$
\begin{aligned}
F_{\nu} \rightarrow F & \text { in } \mathcal{C}^{l}\left(\bar{\Omega} \cap \Delta_{1}, \bigwedge^{0, q}\right), \\
\mathrm{d}_{t} F_{\nu} \rightarrow 0 & \text { in } \mathcal{C}^{l}\left(\Delta_{1}, \bigwedge^{0, q+1}\right)
\end{aligned}
$$

(notice that (4.10) is obtained applying Cauchy's theorem in order to shift the $z$-integral inside $\Omega$ ). If we solve $\mathrm{d}_{t} G_{\nu}=\mathrm{d}_{t} F_{\nu},(\partial / \partial \bar{z}) G_{\nu}=0$ in $\Delta_{1}$, with $G_{\nu} \rightarrow 0$ in $\mathcal{C}^{l}\left(\Delta_{1}, \Lambda^{0, q}\right)$, it follows that $H_{\nu} \doteq F_{\nu}-G_{\nu} \in \mathcal{C}^{l}\left(\Delta_{1}, \bigwedge^{0, q}\right)$ satisfies

$$
(\partial / \partial \bar{z}) H_{\nu}=0, \quad \mathrm{~d}_{t} H_{\nu}=0 \quad \text { in } \Delta_{1}
$$

and

$$
H_{\nu} \rightarrow F \quad \text { in } \mathcal{C}^{l}\left(\bar{\Omega} \cap \Delta_{1}, \bigwedge^{0, q}\right)
$$


Now we solve $\mathrm{d}_{t} g_{\nu}=H_{\nu},(\partial / \partial \bar{z}) g_{\nu}=0$, with $g_{\nu} \in \mathcal{C}^{l}\left(\Delta_{1}, \Lambda^{0, q-1}\right)$. For a fixed $\Delta^{\prime} \in \vartheta$, $\Delta^{\prime} \subset \subset \Delta$, we can find $g_{\nu}^{\prime} \in \mathcal{R}\left(\Delta_{1}, \Lambda^{0, q-1}\right)$ such that

$$
\left\|g_{\nu}-g_{\nu}^{\prime}\right\|_{l, \Delta^{\prime}}<\nu^{-1}
$$

Taking $f_{\nu}=\mathrm{d}_{t} g_{\nu}^{\prime}$ gives our desired conclusion.

(C) We can now easily conclude the proof of Theorem 1.1. We first apply Proposition 4.1 in order to obtain $\Delta_{1} \in \vartheta$ and the sequence $\left\{U_{l}\right\}$ as stated. Then we apply Proposition 4.2: there are $\Delta^{\prime} \in \vartheta, \Delta^{\prime} \subset \Delta_{1}$ and a sequence $f_{l} \in \mathrm{d}_{t} \mathcal{R}\left(\bar{\Omega} \cap \Delta^{\prime}, \Lambda^{0, q-1}\right)$ such that

$$
\left\|U_{l+1}-U_{l}-f_{l}\right\|_{l-1, \bar{\Omega} \cap \Delta^{\prime}}<2^{-l}, \quad l \geqslant 2 .
$$

It follows from (4.15) that the series

$$
U_{2}+\sum_{l=2}^{\infty}\left[U_{l+1}-U_{l}-f_{l}\right]
$$

converges in $\mathcal{R}\left(\bar{\Omega} \cap \Delta^{\prime}, \Lambda^{0, q}\right)$ to a solution of the equation $\mathrm{d}_{t} U=F$.

\section{Appendix: General remarks on the slice condition}

In this appendix we return to the notation established in $\S 1$. In particular, for an open and bounded set $\Omega \subset \mathbf{C} \times \mathbf{R}^{n}$, we shall consider the complex of differential operators between Fréchet spaces

$$
\mathrm{d}_{t}: \mathcal{C}^{\infty}\left(\Omega ; \bigwedge^{0, p}\right) \rightarrow \mathcal{C}^{\infty}\left(\Omega ; \bigwedge^{0, p+1}\right)
$$

and analyze its cohomology in some of its aspects.

As before we let $\pi: \mathbf{C} \times \mathbf{R}^{n} \rightarrow \mathbf{C}$ denote the natural projection and $Y \doteq \pi(\Omega)$; for $z \in Y$ we set $\Omega^{z}=\pi^{-1}\{z\} \cap \Omega=\{z\} \times \Omega(z)$. Recalling that for an open subset $U$ of $\mathbf{R}^{n}$ and for $1 \leqslant r \leqslant n-1$ we have

$$
H^{r}(U, \mathbf{C}) \simeq\left(H_{c}^{n-r}(U, \mathbf{C})\right)^{*}
$$

(de Rham's isomorphism), we conclude that both conditions stated below are equivalent:

$$
\begin{aligned}
H^{r}\left(\Omega^{z}, \mathbf{C}\right)=0 & \text { for all } z \in Y, \\
H_{c}^{n-r}\left(\Omega^{z}, \mathbf{C}\right)=0 & \text { for all } z \in Y .
\end{aligned}
$$


Proposition A.1. Condition (A.2) is equivalent to the following property:

(A.3) To every $\mu \in \mathcal{E}^{\prime}\left(\Omega, \Lambda^{0, n-r}\right)$ satisfying $\mathrm{d}_{t} \mu=0$ there is $\beta \in \mathcal{E}^{\prime}\left(\Omega, \Lambda^{0, n-r-1}\right)$ such that $\mathrm{d}_{t} \beta=\mu$.

Proof. Let $\underline{\mathcal{D}}^{\prime(0, q)}$ denote the sheaf of germs of currents of the kind

$$
w=\sum_{|J|=q} w_{J}(z, t) \mathrm{d} t_{J}, \quad w_{J} \in \mathcal{D}^{\prime}
$$

and let $\mathcal{F}$ denote the sheaf of germs of distributions on $\mathbf{C} \times \mathbf{R}^{n}$ that are independent of $t$. By considering the fine resolution of $\mathcal{F}$,

$$
0 \rightarrow \mathcal{F} \rightarrow \underline{\mathcal{D}}^{\prime(0,0)} \stackrel{\mathrm{d}_{t}}{\longrightarrow} \underline{\mathcal{D}}^{\prime(0,1)} \stackrel{\mathrm{d}_{t}}{\rightarrow} \ldots
$$

then (A.3) is equivalent to

$$
H_{c}^{n-r}(\Omega, \mathcal{F})=0 .
$$

Consider the Leray sheaves $\mathcal{H}_{c}^{q}(\pi, \mathcal{F})$. These are fine sheaves on $Y$, and an elementary application of the Leray spectral sequence (cf. [Br, IV.6]) gives

$$
H_{c}^{n-r}(\Omega, \mathcal{F}) \simeq H_{c}^{0}\left(Y, \mathcal{H}_{c}^{n-r}(\pi, \mathcal{F})\right)
$$

Thus

$$
H_{c}^{n-r}(\Omega, \mathcal{F})=0 \Leftrightarrow \mathcal{H}_{c}^{n-r}(\pi, \mathcal{F})=0 .
$$

Next we make use of the Universal Coefficient Theorem (cf. [Br, II.15]) to conclude that, for all $z \in Y$, the stalk of the Leray sheaf $\mathcal{H}_{c}^{n-r}(\pi, \mathcal{F})$ at $z$ is isomorphic to

$$
H_{c}^{n-r}\left(\Omega^{z}, \mathcal{D}_{z}^{\prime}\right) \simeq H_{c}^{n-r}\left(\Omega^{z}, \mathbf{C}\right) \otimes \mathcal{D}_{z}^{\prime} .
$$

Consequently

$$
H_{c}^{n-r}(\Omega, \mathcal{F})=0 \Leftrightarrow H_{c}^{n-r}\left(\Omega^{z}, \mathbf{C}\right)=0 \quad \text { for all } z \in Y,
$$

which completes the proof.

Let us set

$$
Z^{0, r}(\Omega)=\left\{f \in \mathcal{C}^{\infty}\left(\Omega, \bigwedge^{0, r}\right): \mathrm{d}_{t} f=0\right\}
$$

From Proposition A.1 we obtain 
COROLlaRY A.1. Condition (A.2) is equivalent to the conjunction of the following properties:

(I) $\mathrm{d}_{t}\left\{\mathcal{C}_{c}^{\infty}\left(\Omega, \Lambda^{0, r-1}\right)\right\}$ is dense in $Z^{0, r}(\Omega)$,

(II) $\mathrm{d}_{t}: \mathcal{C}^{\infty}\left(\Omega, \bigwedge^{0, r}\right) \rightarrow \mathcal{C}^{\infty}\left(\Omega, \bigwedge^{0, r+1}\right)$ has closed image.

Proof. (A.2) $\Rightarrow(\mathrm{I})$ : By the Hahn-Banach theorem it suffices to show that if $\mu \in$ $\mathcal{E}^{\prime}\left(\Omega ; \Lambda^{0, n-r}\right)$ satisfies $\mathrm{d}_{t} \mu=0$ then $\mu(g)=0$ for every $g \in Z^{0, r}(\Omega)$, which is of course a consequence of Proposition A.1.

$($ A.2) $\Rightarrow(\mathrm{II})$ : This follows from Proposition A.1 by taking into account the Banach theorem.

Conversely let us assume that (II) holds. Then again by the Banach theorem we derive

$$
\operatorname{Im}\left\{\mathrm{d}_{t}: \mathcal{E}^{\prime}\left(\Omega, \bigwedge^{0, n-r-1}\right) \rightarrow \mathcal{E}^{\prime}\left(\Omega, \bigwedge^{0, n-r}\right)\right\}=Z^{0, r}(\Omega)^{\circ} .
$$

But (I) implies that $Z^{0, r}(\Omega)^{\circ}=\left\{\mu \in \mathcal{E}^{\prime}\left(\Omega, \bigwedge^{0, n-r}\right): \mathrm{d}_{t} \mu=0\right\}$. Thus (A.2) holds and the proof is complete.

We conclude by describing the approach followed in [MT], paying however no attention to estimates. In order to verify the Treves conjecture we must verify, roughly, that for an open and bounded subset $\Omega$ of $\mathbf{C} \times \mathbf{R}^{n}$ which is $(\partial / \partial \bar{z}$ )-convex and satisfies (A.2) for $r=q-1$, the map

$$
\mathrm{d}_{t}: \mathcal{R}\left(\Omega, \bigwedge^{0, q-1}\right) \rightarrow \mathcal{R}\left(\Omega, \bigwedge^{0, q}\right)
$$

has closed image.

Let $f$ belong to its closure. From property (II) in Corollary A.1 we obtain that $f=\mathrm{d}_{t} v$ for some $v \in \mathcal{C}^{\infty}\left(\Omega, \Lambda^{0, q-1}\right)$. We have $\mathrm{d}_{t}(\partial / \partial \bar{z}) v=(\partial / \partial \bar{z}) f=0$, and applying part (I) of Corollary A.1 we conclude that $(\partial / \partial \bar{z}) v$ belongs to the closure of $\mathrm{d}_{t}\left\{\mathcal{C}_{c}^{\infty}\left(\Omega, \Lambda^{0, q-2}\right)\right\}$. If we assume the additional hypothesis that

$$
\mathrm{d}_{t}: \mathcal{C}^{\infty}\left(\Omega, \bigwedge^{0, q-2}\right) \rightarrow \mathcal{C}^{\infty}\left(\Omega, \bigwedge^{0, q-1}\right)
$$

has also closed image (a property that is implied by the validity of (A.2) for $r=q-2$ ) then we would be able to solve

$$
\mathrm{d}_{t} \alpha=(\partial / \partial \bar{z}) v
$$

and consequently $u \doteq v-\mathrm{d}_{t} \beta$, where $\beta \in \mathcal{C}^{\infty}\left(\Omega, \Lambda^{0, q-2}\right)$ satisfies $(\partial / \partial \bar{z}) \beta=\alpha$, would belong to $\mathcal{R}\left(\Omega, \Lambda^{0, q-1}\right)$ and would satisfy $\mathrm{d}_{t} u=f$.

This is the sketch of the argument in [MT]; it is now clear why both conditions $(*)_{q-1}^{+}$ and $(*)_{q-2}^{+}$must be imposed in order to obtain, with such an approach, a solvability theorem in degree $q$ when $q \geqslant 2$. 


\section{References}

[Br] Bredon, G.E., Sheaf Theory, 2nd edition. Graduate Texts in Math., 170. SpringerVerlag, New York, 1997.

[BT] Baouendi, M.S. \& Treves, F., A property of the functions and distributions annihilated by a locally integrable system of complex vector fields. Ann. of Math. (2), 113 (1981), 387-421.

[ChT] Chanillo, S. \& Treves, F., Local exactness in a class of differential complexes. J. Amer. Math. Soc., 10 (1997), 393-426.

[CH1] Cordaro, P.D. \& Hounie, J., On local solvability of underdetermined systems of vector fields. Amer. J. Math., 112 (1990), 243-270.

[CH2] - Local solvability for top degree forms in a class of systems of vector fields. Amer. $J$. Math., 121 (1999), 487-495.

[CT1] Cordaro, P. D. \& Treves, F., Homology and cohomology in hypo-analytic structures of the hypersurface type. J. Geom. Anal., 1 (1991), 39-70.

[CT2] - Hyperfunctions on Hypo-Analytic Manifolds. Ann. of Math. Stud., 136. Princeton Univ. Press, Princeton, NJ, 1994.

[CT3] - Necessary and sufficient conditions for the local solvability in hyperfunctions of a class of systems of complex vector fields. Invent. Math., 120 (1995), 339-360.

[H] Hörmander, L., The Analysis of Linear Partial Differential Operators, IV. Fourier Integral Operators. Grundlehren Math. Wiss., 275. Springer-Verlag, Berlin, 1985.

[MT] MendozA, G.A. \& TREves, F., Local solvability in a class of overdetermined systems of linear PDE. Duke Math. J., 63 (1991), 355-377.

[NT] Nirenberg, L. \& Treves, F., Solvability of a first order linear partial differential equation. Comm. Pure Appl. Math., 16 (1963), 331-351.

[S] Stein, E. M., Singular Integrals and Differentiability Properties of Functions. Princeton Math. Ser., 30. Princeton Univ. Press, Princeton, NJ, 1970.

[T1] Treves, F., On the local solvability and local integrability of systems of vector fields. Acta Math., 151 (1983), 1-38.

[T2] - Hypo-Analytic Structures. Local Theory. Princeton Math. Ser., 40. Princeton Univ. Press, Princeton, NJ, 1992.

Paulo D. Cordaro

Department of Mathematics

Institute of Mathematics and Statistics (IME)

University of São Paulo

Caixa Postal 66281

São Paulo, SP, 05315-970

Brazil

cordaro@ime.usp.br

Jorge G. Hounie

Department of Mathematics

Federal University of São Carlos

São Carlos, SP, 13565-905

Brazil

hounie@power.ufscar.br

Received June 14, 2000 